\title{
Recombinant Human Tumor Necrosis Factor-Related Apoptosis-Inducing Ligand Selectively Induces Apoptosis in Malignant Melanoma
}

\author{
Katherine A. Turner ${ }^{1,2}$, Daniel J. Lindner ${ }^{3}$, and Michael Kalafatis ${ }^{1,2,3,4 *}$ \\ ${ }^{1}$ Department of Chemistry, Cleveland State University \\ ${ }^{2}$ Center for Gene Regulation in Health and Disease (GRHD) \\ ${ }^{3}$ Taussig Cancer Institute, Cleveland Clinic Foundation \\ ${ }^{4}$ Department of Molecular Cardiology, Lerner Research Institute, Cleveland Clinic Foundation
}

*Corresponding author: Michael Kalafatis, Cleveland State University, Department of Chemistry, 2351 Euclid Avenue, Science and Research Center SR370, Cleveland, Ohio 44115, Tel: (216)687-2460; Fax: (216) 687-9298; E-mail: m.kalafatis@csuohio.edu

\begin{abstract}
Skin cancer is among the most commonly-diagnosed cancers with malignant melanoma being associated with the highest rate of metastasis and death. In its early stage, melanoma is easily cured, but the prognosis associated with metastatic malignant melanoma remains very poor and is one of the most treatment-refractory malignancies. This work was undertaken to assess the effectiveness and safety of recombinant human Tumor Necrosis Factor-Related Apoptosis-Inducing Ligand (rhTRAIL) as a potential therapeutic for malignant melanoma. rhTRAIL is the optimized version of the naturally-occurring death-ligand TRAIL. TRAIL shows cancer cell specificity through its innate ability to induce apoptosis in a broad range of transformed human cells while showing no toxicity toward normal healthy cells. Utilizing malignant melanoma A375 cells and normal human melanocytes, the efficacy and safety of rhTRAIL was determined in vitro and in vivo through nude mice A375 xenografts. rhTRAIL induced significant levels of apoptosis in malignant melanoma cells in vitro and at the same time did not induce apoptosis in non-transformed melanocytes. rhTRAIL showed remarkable in vivo potency and was able to inhibit the growth of established melanoma tumors while showing no toxicity towards the mice model. These data suggest that rhTRAIL is a valid candidate for the treatment of malignant melanoma, displaying significant anti-tumor activity with sustainably less negative side effects than traditional therapies.
\end{abstract}

Received Date: October 28, 2016

Accepted Date: January 10, 2017

Published Date: January 17, 2017

Citation: Kalafatis, M., et al. Recombinant Human Tumor Necrosis Factor-Related Apoptosis-Inducing Ligand Selectively Induces Apoptosis in Malignant Melanoma. (2017) Int J Cancer Oncol 4(1): 186- 193.

DOI: $10.15436 / 2377-0902.17 .1191$

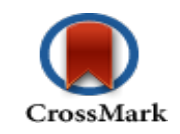

\section{Introduction}

Malignant melanoma is the most deadly form of skin cancer with increasing incidence and mortality worldwide. In its early stage, melanoma has an excellent prognosis, but advanced metastatic melanoma correlates with therapeutic resistance and low survival rates ${ }^{[1,2]}$. Extensive research has resulted in the generation of targeted treatments and immunotherapies for malignant melanoma. However, their utilization is linked with numerous negative side effects, slow effectiveness and only transient effects due to acquired resistance. A standard first-line therapy for malignant melanoma has not yet been established and remains one of the most treatment-refractory malignancies ${ }^{[3,4]}$.
New therapeutic strategies are needed to improve the treatment outcome and survival of malignant melanoma patients.

Several members of the Tumor Necrosis Factor (TNF) family, including FasL, TNF- $\alpha$ and TNF-Related Apoptosis-Inducing Ligand (TRAIL), robustly induce apoptosis in transformed cancer cells ${ }^{[5]}$. However, the therapeutic potential of FasL and TNF- $\alpha$ is hindered by their toxicity upon systemic administration. In contrast, TRAIL selectively induces apoptosis in cancer cells. Even at supraphysiological concentrations, TRAIL shows minimal toxicity to healthy non-transformed cells ${ }^{[6,7]}$. TRAIL acts as a pro-apoptotic ligand through its interactions with extracellular death receptors (DR) DR4 and DR5 ${ }^{[8]}$. Bind- 
ing of TRAIL to DR4 and/or DR5 initiates the extrinsic pathway of apoptosis characterized by the cleavage of procaspase 8 to caspase 8 followed by the activation of downstream executioner caspases 3,6 and 7. TRAIL can also indirectly activate the intrinsic apoptotic pathway through the caspase 8-mediated cleavage of Bid to truncated Bid (tBid). tBid then stimulates the mitochondrial release of cytochrome $\mathrm{c}$ resulting in activation of caspase 9 followed caspases 3, 6 and 7 and the hallmark events of apoptosis ${ }^{[9,10]}$.

In the present study we test a recombinant version of TRAIL, recombinant human TRAIL (rhTRAIL). An optimized form of the apoptosis-inducing portion of the protein, rhTRAIL consists of the extracellular C-domain amino acids 114-281 and lacks any exogenous sequence tags. Clinical trials performed with rhTRAIL demonstrate the safety and tolerability of rhTRAIL. Adverse effects included fatigue, nausea and vomiting, but overall, the effects were mild and well tolerated. The half-life of rhTRAIL was 0.56-1.02 hours with no drug accumulation, antibody formation or hepatotoxicity after receiving multiple doses. The anti-tumorigenic effects of rhTRAIL as a single agent were limited when given to patients with advanced cancer. Few patients showed partial tumor regression while most patients experienced disease progression ${ }^{[11,12]}$. However, when rhTRAIL was given in combination with other anti-cancer therapies the majority of patients responded with complete tumor regression ${ }^{[13,14]}$.

Herein we assessed the potential of rhTRAIL for the treatment of malignant melanoma. With no standard protocol for the treatment of malignant melanoma, rhTRAIL is an excellent candidate due to its selective toxicity towards cancerous cells only. Thus treatment with rhTRAIL will result in maximum anti-tumor effects with decreased negative impact on patients. We aimed to determine the effectiveness of rhTRAIL to act as a cancer cell-specific pro-apoptotic molecule in vitro utilizing malignant melanoma A375 cells and human melanocytes. A375 xenografts were employed to test the in vivo efficacy of rhTRAIL to inhibit the growth of established tumors while showing no toxicity towards the mice model. Overall, these data show the potential of rhTRAIL as a viable candidate for the treatment of malignant melanoma.

\section{Results}

\section{rhTRAIL sensitivity in vitro.}

Malignant melanoma A375 cells and adult human melanocytes were tested for their sensitivity to rhTRAIL-induced apoptosis in vitro. Cells were treated with increasing concentrations of rhTRAIL ranging from $5 \mathrm{ng} / \mathrm{ml}$ to $1 \mu \mathrm{g} / \mathrm{ml}$ for 72 hours in full medium. Levels of rhTRAIL-induced apoptosis were determined by FITC-Annexin-V and PI staining followed by FACS analysis. A375 cells showed a dose-dependent induction of apoptosis in response to rhTRAIL marked by the increasing formation of Annexin- $\mathrm{V}^{+}$and/or Annexin- $\mathrm{V}^{+}$and $\mathrm{PI}^{+}$cells (Figure. 1A \& B). rhTRAIL was able to induce apoptosis even at the lowest tested rhTRAIL concentration of $5 \mathrm{ng} / \mathrm{ml}$ with 9.7 $\pm 1.0 \%$ apoptotic cells $(\mathrm{P}<0.02)$, increasing to $44.6 \pm 0.5 \%$ apoptotic cells $(\mathrm{P}<0.02)$ in the highest treated group of $1 \mu \mathrm{g} / \mathrm{ml}$. In contrast, human melanocytes were completely insensitive to rhTRAIL-induced apoptosis (Figure. 1A \& C). Treatment with rhTRAIL, even at the highest tested treatment concentration of
$1 \mu \mathrm{g} / \mathrm{ml}$ of rhTRAIL, did not result in the formation of apoptotic cells as indicated by the lack of Annexin- $\mathrm{V}^{+}$and/or Annexin- $\mathrm{V}^{+}$ and $\mathrm{PI}^{+}$cells as compared to the control $(\mathrm{P}>0.05)$.

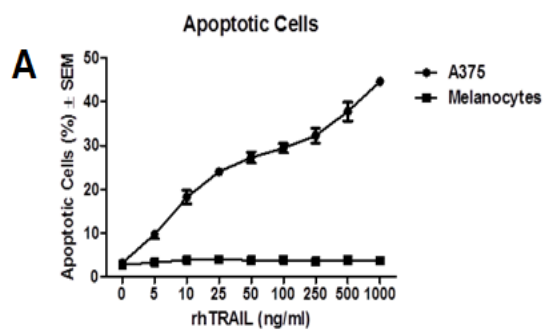

B
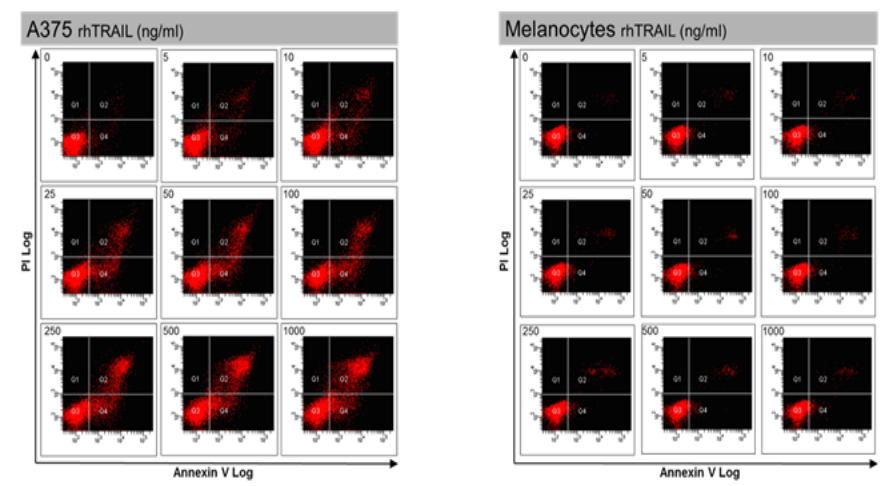

Figure 1: rhTRAIL sensitivity in vitro. Sensitivity of A375 and melanocytes to rhTRAIL-induced apoptosis.

A) Average of three independent assays \pm SEM. B) Representative histogram of A375. C) Representative histogram of melanocytes. Lower left quadrant: Viable cells (Annexin-/PI-), Lower right quadrant: Early apoptotic cells (Annexin $\left.{ }^{+} / \mathrm{PI}^{-}\right)$, Upper right quadrant: Late apoptotic cells $\left(\right.$ Annexin $\left.{ }^{+} / \mathrm{PI}^{+}\right)$.

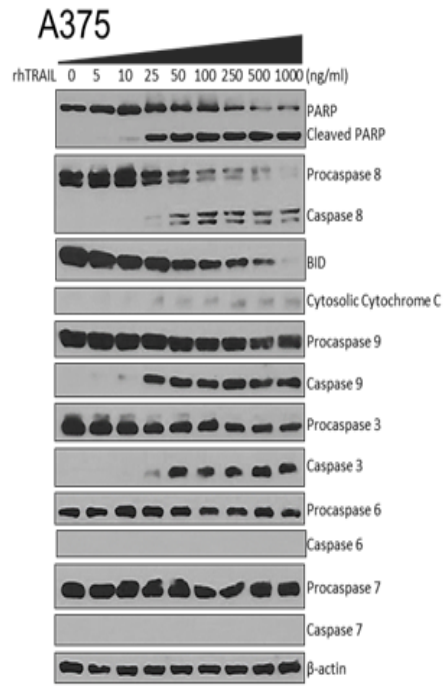

A

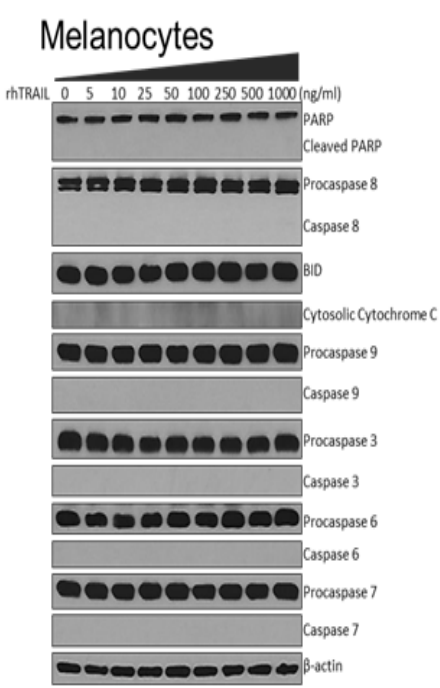

B
Figure 2: Western blot analysis of apoptosis-related proteins. A) A375 and B) Melanocytes \pm rhTRAIL subjected to western blot analysis and probed with anti-PARP, caspase 8 , Bid, cytochrome c, caspase 9 , caspase 3 , caspase 6 and caspase 7 . $\beta$-actin was used as a loading control for each membrane. Representative $\beta$-actin is depicted. Western blots were done in duplicates.

\section{Mechanism of rhTRAIL-initiated apoptosis}

To further examine the pathway of apoptosis induced 
by rhTRAIL western blotting was employed using antibodies to various components of the apoptosis cascade. As described above A375 cells and human melanocytes were treated with increasing concentrations of rhTRAIL for 72 hours, collected and whole cell lysates were resolved by SDS-PAGE and subjected to western blotting. rhTRAIL significantly induced apoptosis in A375 noted by the fragmentation of the DNA repair enzyme Poly-(ADP) Ribose Polymerase (PARP), starting at $10 \mathrm{ng} / \mathrm{ml}$ rhTRAIL (Figure. 2A). Treatment with rhTRAIL was able to effectively initiate the extrinsic pathway of apoptosis in A375 as indicated by the cleavage of procaspase 8 to caspase 8 . Additionally, rhTRAIL was able to indirectly initiate the intrinsic (mitochondrial) pathway of apoptosis through the caspase 8-mediated cleavage of $\mathrm{Bid}$, as demonstrated by the release of cytochrome c from the mitochondria into the cytosol and the activation of caspase 9. Lastly, executioner caspase 3 was activated, whereas, other executioner caspases 6 and 7 were not activated through treatment with rhTRAIL. Adult human melanocytes were completely resistant to rhTRAIL-induced apoptosis and did not show PARP cleavage or activation of any other proteins in the apoptotic pathways (Figure. 2B).

\section{Death Receptor Expression}

To explain the mechanism of rhTRAIL-selectivity, the membrane expression of DR4 and DR5 were measured on A375 cells and melanocytes through staining with fluorescent antibodies to DR4 and DR5 followed by FACS analysis. Overall, malignant A375 cells had significantly higher expression of both DR4 and DR5 (Figure. 3). Comparing the membrane expression of DR4, A375 cells had significantly higher levels with a mean fluorescence intensity (MFI) of $70.3 \pm 7.3$ compared to melanocytes with a MFI of $33.3 \pm 1.5(\mathrm{P}<0.01)$ (Figure. 3A). For DR5,
A375 cells had a higher level of membrane expression with a MFI of $1591.3 \pm 66.9$ compared with melanocytes with a MFI of $758.0 \pm 7.5(\mathrm{P}<0.01)$ (Figure. 3B).

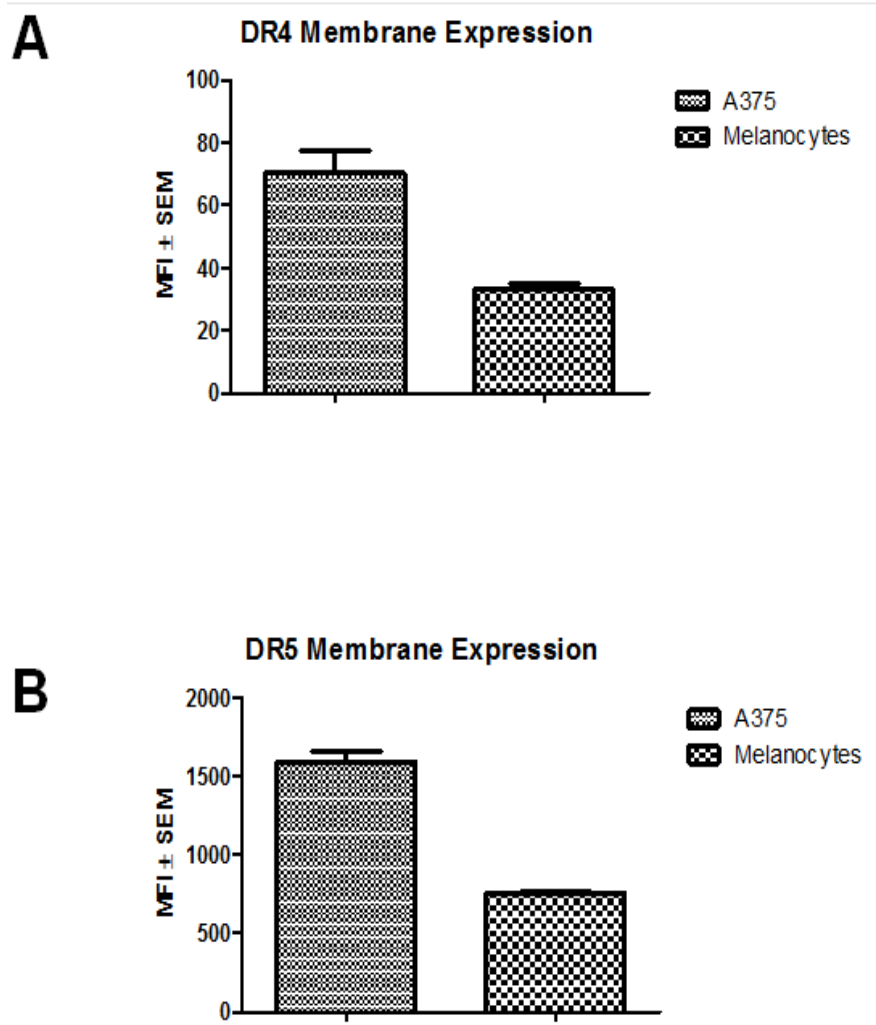

Figure 3: Death Receptor Expression. Membrane expression of rhTRAIL-binding receptors DR4 and DR5. Mean Fluorescent Intensity (MFI) \pm SEM. Average of three independent assays. A) A375 B) Melanocytes.
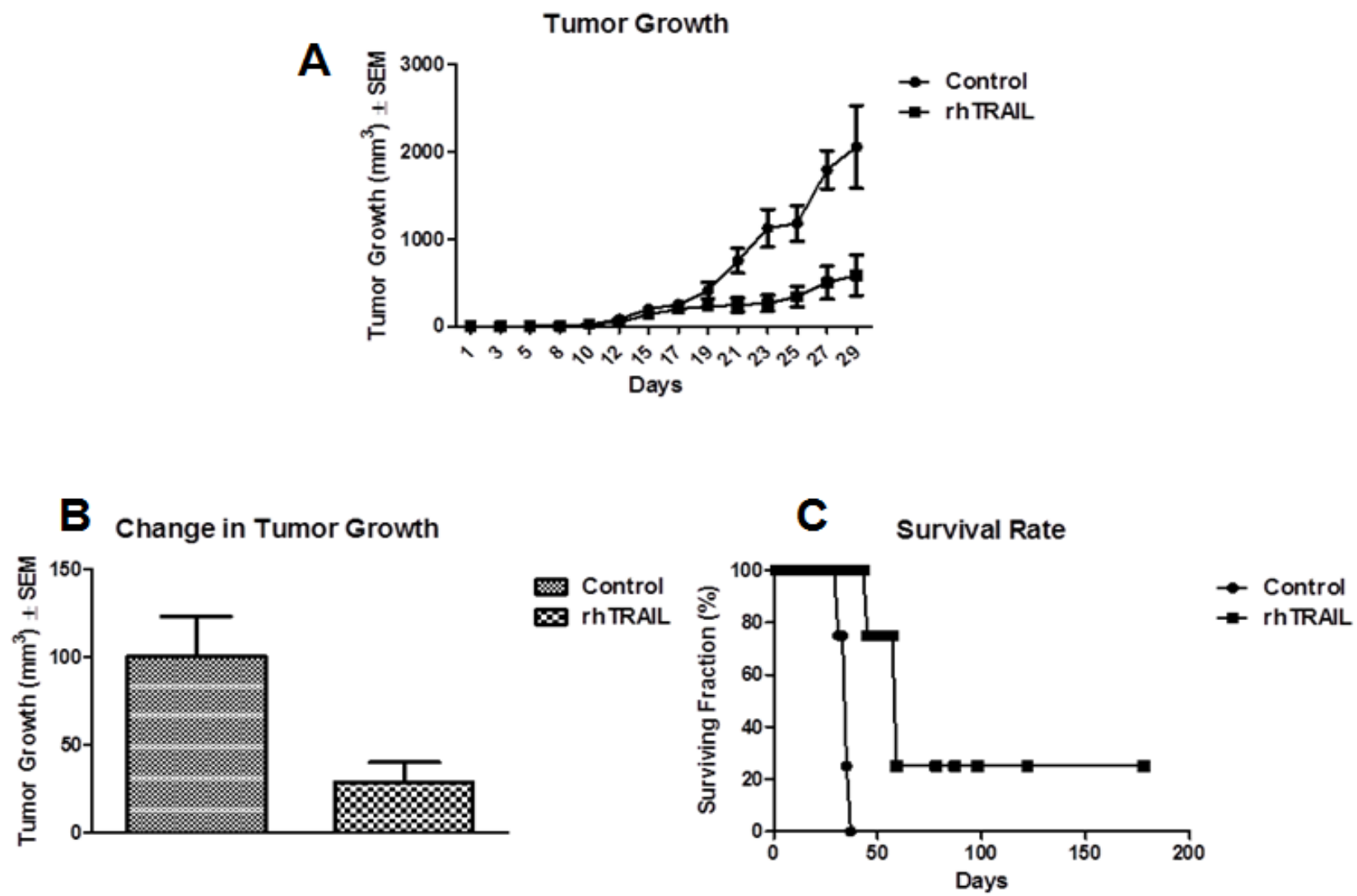

Figure 4: Anti-tumor activity of rhTRAIL. Sensitivity of A375 to rhTRAIL in vivo. Treatment began day 18 . rhTRAIL-mice received $32 \mathrm{mg} / \mathrm{kg}$ and control mice received PBS ip for five days on a 21-day cycle. A) Tumor growth curve. B) End point tumor volume. C) Survival rate. 


\section{Anti-tumorigenic effects of rhTRAIL in vivo}

To test the in vivo efficacy of rhTRAIL we further employed nude mice xenografts. Female athymic nu/nu mice were inoculated with $1.6 \times 10^{6} \mathrm{~A} 375$ cells subcutaneously in both flanks and allowed to grow established tumors $\left(\sim 200 \mathrm{~mm}^{3}\right)$. Treatment began on day 18 with the rhTRAIL-treated group $(\mathrm{n}=$ 8) receiving $32 \mathrm{mg} / \mathrm{kg}$ rhTRAIL for five days on a 21-day cycle. Control mice $(n=8)$ received the vehicle of PBS on the same schedule. All mice were supplemented with $100 \mathrm{mg} / 1 \mathrm{ZnCl}_{2}$ in the water daily and tumor volume was measured three times a week. At the death of the first mouse in the control group at day 29 , mice treated with rhTRAIL had an average tumor size of $585.9 \pm 232.6 \mathrm{~mm}^{3}$ while the control mice had an average tumor size of $2055.3 \pm 476.8 \mathrm{~mm}^{3}$ (Figure. 4A). rhTRAIL-treated mice had $71.5 \%$ less tumor growth than the vehicle-treated controls ( $\mathrm{P}$ $<0.02$ ) (Figure. 4B). Additionally, the survival rate of the mice was significantly enhanced following treatment with rhTRAIL compared to the control (Figure. 4C). For the control group, all mice had to be sacrificed by day 35 due to extremely large tumor size; however, in the rhTRAIL group $100 \%$ of the mice survived to day $43,75 \%$ to day 57 and one mouse lived until day 178 . As compared to the control, rhTRAIL-treated mice maintained their weight, activity and exhibited no adverse side effects as determined by visual inspection.

\section{Xenograft histology}

Tumors and major organs of sacrificed mice were collected and analyzed. TUNEL staining of the tumors revealed significant induction of apoptosis in the rhTRAIL-treated tumors compared to the control mice (Figure. 5A \& B). Control tumors had $0.3 \pm 0.1 \%$ TUNEL positive cells relative to DAPI stained cells compared to the rhTRAIL-treated tumors which had 52.3 $\pm 5.7 \%$ TUNEL positive cells relative to DAPI stained cells (P $<0.00001)$. H\&E staining was also performed on the harvested tumors (Figure. 5C). rhTRAIL-treated tumors showed signs of apoptosis evidenced by the presence of irregularly shaped and condensed nuclei compared to the control tumors possessing nicely defined nuclei. Finally, major organs, liver, kidney and spleen, were harvested and analyzed by H\&E staining (Figure. 5C). Compared to the control, the rhTRAIL-treated organs were healthy and showed no signs of any rhTRAIL-induced toxicity.
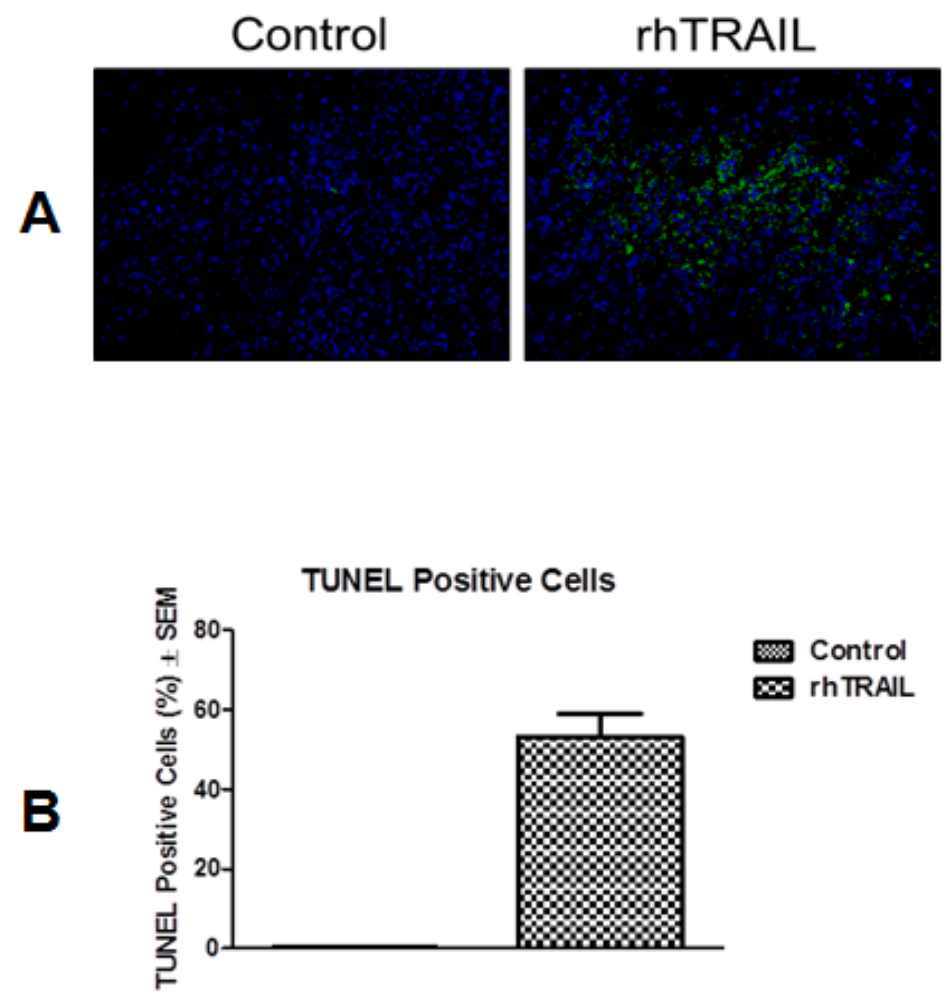

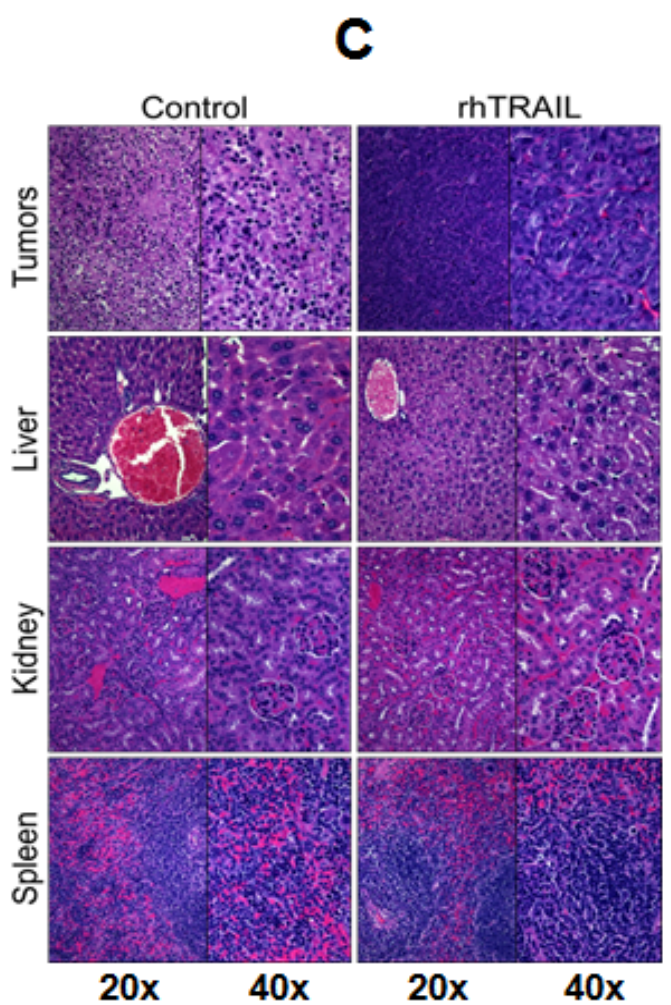

Figure 5: Xenograft tumor and organ analysis. A) TUNEL staining of harvested tumors. Representative picture, 20x. B) Percent TUNEL positive cells relative to DAPI positive cells \pm SEM. Values represent the average of three tumors. C) H\&E staining of tumors and major organs. Representative pictures, 20x and 40x.

\section{Discussion}

rhTRAIL shows great promise as a pro-apoptotic anti-cancer therapeutic, possessing the ability to selectively target and induce apoptosis in a wide variety of human cancers ranging from solid tumors such as colon, lung and breast to hematological cancers such as leukemia and lymphoma. At the same time, rhTRAIL does not activate apoptosis in normal cells including endothelial, astrocytes and hematopoietic stem cells ${ }^{[15,16]}$. Currently, advanced cases of malignant melanoma are one of the leading causes of death worldwide with no effective treatment option. Here we analyze rhTRAIL as a potential anti-melanoma therapeutic. These data shows that rhTRAIL is highly efficient in selectively killing malignant melanoma cells while demonstrating no toxicity towards non-transformed melanocytes. The hallmark events of apoptosis, generation of apoptotic cells (Annexin- $\left.\mathrm{V}^{+}\right)^{[17]}$ and the fragmentation of the DNA repair enzyme PARP $^{[18]}$, occurred in A375 cells but not in normal melanocytes. rhTRAIL initiates the extrinsic pathway of apoptosis through the binding of DR4 and DR5 ${ }^{[19]}$. We show that rhTRAIL is extremely 
effective in directly activating the extrinsic pathway of apoptosis apparent by the robust activation of caspase 8 in melanoma cells but not in normal melanocytes. At the highest dose of rhTRAIL nearly all procaspase 8 is converted to caspase 8 , revealing the high affinity of rhTRAIL for DR4 and DR5 in melanoma cells. The difference in rhTRAIL-sensitivity between cancer cells and normal cells may lie in their membrane expression of $\mathrm{DRs}^{[20,21]}$. Examination of the membrane expression of DR4 and DR5 showed that melanocytes have 2-fold less membrane expression of both DR4 and DR5 as compared to malignant melanoma A375 cells. The decreased expression of DRs may explain why rhTRAIL does not harm normal cells and is well suited and safe for systemic administration as an anti-cancer therapeutic with low side effects for patients.

There is crosstalk between the extrinsic and intrinsic pathways of apoptosis mediated by the cleavage of Bid to tBid. tBid translocates to the mitochondria and initiates the intrinsic pathway by promoting the release of cytochrome $\mathrm{c}$ into the cytosol. This mitochondrial amplification loop intensifies the pro-apoptotic signal initiated by the rhTRAIL-activated extrinsic pathway, resulting in enhanced apoptosis compared to either pathway alone ${ }^{[22]}$. Initiation of the intrinsic pathway typically occurs through activation of p53 in response to cellular stresses such as DNA damage. This is the mechanism by which many chemotherapeutic agents and ionizing radiation kill malignant cells. However, in over $50 \%$ of all cancers, p53 is mutated and resistance to standard p53-dependent cancer therapies often occurs $^{[23]}$. As a result of defective p53 in cancerous cells, traditional therapies often result in severe systemic toxicity by destroying normal cells possessing the functional form of p53, causing numerous side effects that negatively influence the patient's quality of life and prevent optimal drug-dosing ${ }^{[24]}$. However, we show that rhTRAIL can indirectly activate the intrinsic pathway of apoptosis and bypass the need for p53. Therefore, the application of rhTRAIL for the treatment of cancer can be used for any tumor type regardless of its p53 status. Additionally, the anti-tumorigenic effects of rhTRAIL are superior over traditional therapies due to the potential activation of both the extrinsic and intrinsic pathways of apoptosis with significantly less negative impact on the patient.

In clinical trials, patients with advanced cancers were treated with rhTRAIL alone or in combination with other therapies such as Rituximab in non-Hodgkin's lymphoma or in combination with Paclitaxel, Carboplatin and Bevacizumab (PCB) in advanced non-squamous non-small-cell lung cancer (NSCLC). rhTRAIL was given at doses ranging from $0.5-35.0 \mathrm{mg} /$ $\mathrm{kg}$ I.V. for five days on a 21-day cycle ${ }^{[11-14]}$. To test the in vivo efficacy of rhTRAIL, nude mice A375 xenografts modeled after clinical trials were employed and mice were treated with $32 \mathrm{mg} /$ $\mathrm{kg}$ rhTRAIL ip for five days on a 21-day cycle. Crystallographic studies show that TRAIL has a homotrimeric structure, consisting of three TRAIL monomers coordinated by an internal zinc atom. The zinc atom is buried at the center of the trimer, coordinated by a single cysteine residue on each monomer and is required for maintaining the structure, stability and the overall biological activity of TRAIL. Reports indicate that preparations of rhTRAIL lacking adequate zinc concentrations are associated with decreased apoptotic activity ${ }^{[25,26]}$. To enhance the bioactivity of rhTRAIL in vivo, $\mathrm{ZnCl}_{2}$ was continuously provided in the drinking water of the mice. It has been repetitively demon- strated that Zinc is necessary and required for optimal TRAIL physiological function. Presented here is a novel methodology of adding of $\mathrm{ZnCl}_{2}$ to the drinking water of mice in a pre-clinical in vivo rhTRAIL experiment. Under these conditions, rhTRAIL showed great in vivo efficacy and was able to inhibit the growth of established tumors while being safe and nontoxic to the mice model. As compared to the controls, rhTRAIL-treated mice had $71.5 \%$ less tumor growth. Upon starting rhTRAIL treatment, tumor growth leveled off; whereas, tumors of control mice grew exponentially over time. The in vivo anti-tumor effects of rhTRAIL where confirmed in a second A375 xenograft experiment in which the drinking water of the mice was also supplemented with $\mathrm{ZnCl}_{2}$. At the death of the first control mouse, rhTRAIL-treated mice had $68.0 \%$ less tumor growth than the control mice $(\mathrm{P}<0.01)$ (data not shown). Additionally, rhTRAIL was able to significantly prolong the survival of the cancer-bearing mice. Specifically, by day 35 all the control mice had to be sacrificed due to extremely large tumor volume. In contrast, $100 \%$ of the mice in the rhTRAIL group survived to day 43 and one mouse lived until day 178. The fact that a mouse lived until day 178 without any further treatment is quite significant because it had large tumors that were eliminated during rhTRAIL-treatment and did not regrow after the treatment had ended.

The mechanism of rhTRAIL-mediated tumor growth inhibition was established in the mice. Intra-tumor apoptosis was confirmed through TUNEL staining which identifies apoptotic cells ${ }^{[27]}$. TUNEL staining revealed significantly high levels of apoptotic cells (TUNEL ${ }^{+}$) in the rhTRAIL-treated tumors compared to the control. Additionally, H\&E staining of the tumors showed irregularly shaped and condensed nuclei in the rhTRAIL-treated tumors, indicative of induction of apoptosis ${ }^{[28]}$. Together, the H\&E and TUNEL staining show that inhibition of tumor growth within the rhTRAIL-treated A375 xenografts was due to rhTRAIL-induced apoptosis. Lastly, upon sacrifice of the mice, major organs, liver, kidney and spleen, were harvested and analyzed by H\&E staining. Compared to the control, rhTRAIL-treated mice showed no signs of systemic toxicity to any of the major organs analyzed. In addition, rhTRAIL-treated mice were active, alert and they maintained their weight as compared to the control. The histological analysis together with the physical appearance of the rhTRAIL-treated mice confirm that although rhTRAIL is extremely effective at inhibiting tumor growth it is still safe and well tolerated upon systemic administration.

The only previously reported rhTRAIL-treated A375 xenograft shows that when treating mice at $50 \mathrm{mg} / \mathrm{kg}$ every other day tumor growth was not inhibited as compared the control ${ }^{[29]}$. However, we show that rhTRAIL is able to significantly suppress tumor growth demonstrating of the superiority of our optimized rhTRAIL molecule. The previous xenograft experiment began their treatment two days after the A375 cells were inoculated and before the establishment of a substantial tumor. Whereas in our experiment, rhTRAIL-treatment began on day 18 when established tumors, approximately $200 \mathrm{~mm}^{3}$ in size, were formed. rhTRAIL was given to mice with established tumors to best model clinical settings and to see if rhTRAIL can eliminate established tumors. In the previous experiment, even at much higher concentrations of rhTRAIL, tumor growth was not impeded and was unable to stop the formation of A375 xe- 
nograft tumors. With our rhTRAIL, we were able to stop the growth of already growing tumors and significantly eliminate the tumor load on the cancer-bearing mice. Additionally, extra zinc was not provided in the drinking water of the mice in the previous experiment. The addition of $\mathrm{ZnCl}_{2}$ may account for the success of the presented A375 xenograft compared to the previous study, since saturating amounts of zinc are needed for the optimal activity of rhTRAIL ${ }^{[25,26]}$. This may reveal the need to supplement patients receiving rhTRAIL with extra zinc to increase the in vivo efficiency of rhTRAIL. Lastly, in the previous experiment, rhTRAIL was given every other day and caused no impact on tumor growth. However, we administered rhTRAIL every day for five days on a 21-day cycle and tumor growth was successfully hindered. Although rhTRAIL only has a half-life of about an hour, it is still able to substantially inhibit tumor growth in rhTRAIL-sensitive cancer cells. The success of our experiment shows the importance of treating everyday with rhTRAIL for it to be most effective.

Although rhTRAIL shows the ability to induce apoptosis in a broad range of human cancers, rhTRAIL-sensitivity is heterogeneous. Some cell lines display resistance to rhTRAIL-induced apoptosis while others can acquire resistance after repeated exposure ${ }^{[30]}$. For example, a study of eight human melanoma cell lines treated with increasing concentrations of rhTRAIL found that five of the lines were sensitive to rhTRAIL, while three lines were resistant ${ }^{[31]}$. Current literature describes a myriad of ways in which sensitivity to rhTRAIL may be controlled, which is often cell-dependent. One mechanism of rhTRAIL-resistance is the increased expression of inhibitor of apoptosis proteins (IAPs) such as FLIP, XIAP, cIAP and survivin $^{[32]}$. Moreover, the equilibrium between pro- and anti-apoptotic members of the Bcl-2 family plays an important role in rhTRAIL-sensitivity. Overexpression of anti-apoptotic proteins Bcl-2 and Bcl-xL correlate with rhTRAIL-resistance along with downregulation of pro-apoptotic proteins Bax and $\mathrm{Bak}^{[33]}$. Finally, rhTRAIL-resistance may occur because of the lack of a optimum concentration of cell surface receptors. rhTRAIL-resistance has been associated with low expression levels or mutations in the rhTRAIL-binding region of the DRs ${ }^{[6]}$. Studies suggest that an increased potency against cancer cells is achieved when rhTRAIL is administered as a combination therapy. An increase in rhTRAIL-induced apoptosis was seen in co-treatments with chemotherapy, radiotherapy, irradiation and even with nontraditional therapies such as proteasome inhibitors, histone deacetylase inhibitors and the tyrosine kinase inhibitor in vitro and in vivo ${ }^{[16]}$. A successful co-treatment is one in which anti-apoptotic proteins such as FLIP and XIAP are downregulated or DRs can be upregulated on the cancer cell surface ${ }^{[34,35]}$. By combining rhTRAIL with compounds that are able to reverse the intrinsic cellular mechanisms of rhTRAIL-resistance, cancer cells that were once resistant to rhTRAIL-induced apoptosis can now be sensitized ${ }^{[36]}$.

rhTRAIL remains an attractive candidate as an anti-cancer therapy because it is able to selectively induce apoptosis in cancer cells while not harming normal healthy cells ${ }^{[37]}$. In the present work we show that rhTRAIL induces apoptosis in vitro and in vivo in malignant melanoma A375 cells. Our data demonstrates that rhTRAIL can induce high levels of apoptosis through direct activation of the extrinsic pathway and the indirect activation of the intrinsic pathway. We have validated the specific- ity and safety of rhTRAIL towards normal cells. rhTRAIL was able to induce robust levels of apoptosis malignant melanoma cells but not in normal adult human melanocytes. These data demonstrate the specificity of rhTRAIL for transformed cells. The ability of rhTRAIL to exhibit significant anti-tumor activity without harming normal cells contrasts traditional chemotherapies where all cells, normal and cancerous, are targeted for cell death ${ }^{[38,39]}$. Therefore, development of anti-cancer agents such as rhTRAIL that specifically target cancer cells is essential to enhance the treatment and overall quality of life for cancer patients. In conclusion, these data show that rhTRAIL would be an effective treatment for malignant melanoma with little potential for negative side effects. Thus, treatment with rhTRAIL would fulfill the need for a potent and safe treatment option for malignant melanoma resulting in maximum anti-tumor effects with decreased negative impact on patients' lives.

\section{Methods}

Drugs and Chemicals: Recombinant human Tumor Necrosis Factor-Related Apoptosis-Inducing Ligand (rhTRAIL) was produced according to well defined and previously detailed protocols $^{[40-42]}$. Briefly, rhTRAIL was produced in E.Coli using an optimized cDNA for the particular strain used. Following induction for $22 \mathrm{~h}$, the cell paste was harvested and rhTRAIL was purified stepwise by FPLC using previously described methods. rhTRAIL was purified to homogeneity and analyzed at each step by SDS-PAGE following staining with Coomassie. The final product was found to be $>99 \%$ pure as demonstrated by HPLC and mass spectrometry.

Cell Culture: Human adult primary epidermal melanocytes (ATCC PCS-200-013) were maintained in Dermal Cell Basal Medium (ATCC PCS-200-030) supplemented with Adult Melanocyte Growth kit (ATCC PCS-200-042) and 1\% Antibiotic/Antimycotic Solution $(10,000 \mathrm{IU} / \mathrm{ml}$ penicillin, $10 \mathrm{mg} / \mathrm{ml}$ streptomycin and $25 \mu \mathrm{g} / \mathrm{ml}$ amphotericin). A375 cells were maintained in DMEM, supplemented with 10\% Fetal Bovine Serum (FBS) and $1 \%$ Antibiotic/Antimycotic Solution. Cells were incubated in a $90 \%$ humidified atmosphere with $5 \% \mathrm{CO}_{2}$ at $37^{\circ} \mathrm{C}$.

Apoptosis Assay: Treated cells were trypsinized, harvested, washed twice with cold PBS and resuspended in $100 \mu \mathrm{l}$ of Annexin- $\mathrm{V}$ binding buffer at a concentration of $1 \times 10^{3}$ cells $/ \mu \mathrm{l}$. According to manufacturer's protocol, cells were incubated with 5 $\mu \mathrm{l}$ of FITC-Annexin-V and Propidium Iodide (PI) for 15 minutes at room temperature in the dark (FITC-Annexin-V Kit Apoptosis Detection Kit I, BD Pharminogen). Stained cells were analyzed on BDFACS Canto II using Diva software. Single color controls (Annexin-V or PI only) were used to set up compensation and quadrants for FACS.

Western Blot Analysis: Total cell lysates were prepared using RIPA lysis buffer (Sigma) containing $150 \mathrm{mM}$ sodium chloride, $1.0 \%$ Triton $\mathrm{X}-100,0.5 \%$ sodium deoxycholate, $0.1 \%$ sodium dodecyl sulfate (SDS) and $50 \mathrm{mM}$ Tris, $\mathrm{pH} 8.0$ plus a $1 \mathrm{x}$ cocktail of protease inhibitors (Protease Inhibitor Cocktail Set I, Calbiochem). Cells were lysed for 30 minutes at $4^{\circ} \mathrm{C}$ followed by centrifugation for 10 minutes at $10,000 \mathrm{rpm}$ at $4^{\circ} \mathrm{C}$. Protein concentrations were determined using BCA protein as- 
say (Pierce). A $35 \mu$ g protein aliquot was mixed with $4 x$ Laemmli's SDS sample buffer (0.02\% Bromophenol Blue (BPB), 8\% Beta-mercaptoethanol (BME), 8\% SDS, 40\% glycerol and $250 \mathrm{mM}$ Tris-HCl, $\mathrm{pH}$ 6.8). Cell lysates were heated for five minutes at $100^{\circ} \mathrm{C}$, resolved by $12 \%$ SDS-polyacrylamide gel electrophoresis (SDS-PAGE) and transferred to polyvinylidene fluoride (PVDF) membrane. The membrane was blocked with $5 \%$ non-fat milk or $5 \%$ BSA for $\geq 1$ hour and incubated with primary antibodies for PARP, caspase 8 , caspase 3 , Bid, caspase 9, caspase 6, caspase 7 (Cell Signaling). After incubation, the membrane was incubated with secondary anti-rabbit or mouse horseradish peroxidase (HRP)-conjugated antibodies (Biorad). Proteins were visualized through development by enhanced chemiluminescence (ECL 2 Western Blotting Substrate, Pierce) and exposure on X-ray film. The blots were reprobed for $\beta$-actin to confirm equal protein loading.

Cytochrome c Release: Cells were resuspended in permeabilization buffer $(400 \mu \mathrm{g} / \mathrm{mL}$ digitonin, $75 \mathrm{mMKCl}, 1 \mathrm{mM}$ $\mathrm{NaH}_{2} \mathrm{PO}_{4}, 8 \mathrm{mM} \mathrm{Na}_{2} \mathrm{HPO}_{4}$ and $250 \mathrm{mM}$ sucrose) plus protease inhibitors. Samples were incubated for 10 minutes at $4^{\circ} \mathrm{C}$, centrifuged at $16,000 \mathrm{~g}$ for five minutes at $4^{\circ} \mathrm{C}$ and the supernatants were kept as the cytosolic fraction. Samples were quantified as described above and $60 \mu \mathrm{g}$ was resolved on a $15 \%$ gel. As described above, the gel was transferred to PVDF, blocked and incubated with anti-cytochrome c (Cell Signaling) and visualized by chemiluminescence.

Death Receptor Membrane Expression: Treated cells were collected with enzyme-free PBS-based cell dissociation buffer (Gibco Life Technologies) and stained with mouse anti-human DR4 and DR5 conjugated to phycoerythrin (PE) (eBioscience). Briefly, $0.25 \times 10^{6}$ cells were incubated in $100 \mu 1$ of staining buffer $(2 \%$ FBS, $0.02 \%$ sodium azide in PBS) and $5 \mu 1$ anti-DR 4 or anti-DR5 for one hour on ice in the dark. As a negative control, cells were stained with mouse $\operatorname{IgG} 1 \kappa$ isotype antibody under the same conditions. After incubation, cells were washed twice with staining buffer and resuspended in $500 \mu 1$ of staining buffer and analyzed on BDFACS Canto II using FACS Diva software.

Xenografts: Animal experiments were performed in accordance with guidelines approved by the Institutional Animal Care and Use Committee (IACUC) at the Cleveland Clinic Foundation. Female athymic nu/nu mice (Taconic Farms, Inc.) supplement with $100 \mathrm{mg} / 1 \mathrm{ZnCl}_{2}$ water were inoculated with $1.6 \times 10^{6} \mathrm{~A} 375$ cells subcutaneously in both flanks. Tumor volume was monitored until tumors reached approximately $200 \mathrm{~mm}^{3}$. Tumor dimensions were measured using calipers and tumor volume was calculated using the formula for a prolate spheroid $\left(V=4 / 3 \pi a^{2} b\right.$, where $\mathrm{a}=$ minor radius and $\mathrm{b}=$ major radius of the tumor) three times a week. Upon formation of established tumors, mice were randomized into groups $(\mathrm{n}=8)$. rhTRAIL-treated mice received $32 \mathrm{mg} / \mathrm{kg}$ for five days on a 21-day cycle and vehicle-treated controls received $250 \mu \mathrm{L}$ PBS on the same cycle intraperitoneally (ip). The physical status of the mice, weight and activity, were visually monitored and treatments continued until the mice reached their end point and were sacrificed in accordance with IACUC.

Immunohistochemistry: Tumors were subjected to TUNEL Staining (ApopTagPlus FloresceinIn Situ Apoptosis Detection
Kit, Millipore) according to manufacturer's protocol. Briefly, tumors were fixed in $10 \%$ formalin, treated with protein kinase $\mathrm{K}$, washed twice with PBS, incubated in equilibration buffer for 10 minutes and incubated with terminal deoxynucleotidyl transferase for 60 minutes at $37^{\circ} \mathrm{C}$. Cells were washed with stop buffer for 10 minutes and then incubated with antidigoxigenin conjugate for 30 minutes at $25^{\circ} \mathrm{C}$. After washing in PBS, cells were mounted with DAPI and viewed on a fluorescent microscope.

Xenograft Histology: Following euthanasia, liver, kidney, spleen, and tumors were harvested and fixed in 10\% neutral buffered formalin. Sections of tumors and organs were stained with $\mathrm{H} \& \mathrm{E}$ and viewed microscopically.

Statistical Analysis: Student t-test was used to determine significance. $P$ values less than 0.05 were deemed significant.

Funding: This work was supported by funds from a grant from the Parker Hannifin Foundation (MK), and by a P30 CA04370322 Case Comprehensive Cancer Center Support Grant (DJL).

Conflicts of Interest: There are no disclaimers or conflicts of interest to disclose.

\section{References}

1. American Cancer Society. Cancer Facts \& Figures (2016) Atlanta: American Cancer Society.

2. Guy, G.P., Thomas, C.C., Thompson, T., et al. Vital signs: melanoma incidence and mortality trends and projections - United States, 19822030. (2015) MMWR Morb Mortal Wkly Rep 64(21): 591-596.

3. Mouawad, R., Sebert, M., Michels, J., et al. Treatment for metastatic malignant melanoma: old drugs and new strategies. (2009) Critical reviews in oncology/hematology 74(1): 27-39.

4. Meric, J., Rixe, O., Khayat D. Metastatic malignant melanoma. (2003) Drugs Today (Barc) 39 Suppl C: 17-38.

5. Ashkenazi, Dixit. Death Receptors: Signaling and Modulation. (1998) Science 281(5381): 1305-1308.

6. Duiker, Mom, de Jong, et al. The clinical trail of TRAIL. (2006) Eur J Cancer. (Oxford, England : 1990) 42(14): 2233-2240.

7. Kimberley, F., Screaton, G. Following a TRAIL: Update on a ligand and its five receptors. (2004) Cell Research 14(5): 359-372.

8. Gaur, U., Aggarwal, B. Regulation of proliferation, survival and apoptosis by members of the TNF superfamily. (2003) Biochem Pharmacol 66(8): 1403-1408.

9. Stuckey, D., Shah, K. TRAIL on trial: preclinical advances in cancer therapy. (2013) Trends Mol Med 19(11): 685-694.

10. Hengartner, M. The biochemistry of apoptosis. (2000) Nature 407(6805): 770-776.

11. Ling, J., Herbst, R.S. Apo2L/TRAIL pharmacokinetics in a phase 1a trial in advanced cancer and lymphoma. (2006) J Clin Oncol 24(18s): 3047.

12. Herbst, R., Eckhardt, G., Kurzrock, R., et al. Phase I Dose-Escalation Study of Recombinant Human Apo2L/TRAIL, a Dual Proapoptotic Receptor Agonist, in Patients with Advanced Cancer. (2010) J Clin Oncol 28(17): 2839-2846.

13. Yee, L., Fanale, M., Dimick, K., et al. A phase IB safety and pharmacokinetic (PK) study of recombinant human Apo2L/TRAIL in combination with rituximab in patients with low-grade non-hodgkin lymphoma. (2007) J Clin Oncol 25: 8078-8112.

14. Soria, J.C., Smit, E., Khayat, D., et al. Phase 1b Study of Dulanermin (recombinant human Apo2L/TRAIL) in Combination With Paclitaxel, Carboplatin, and Bevacizumab in Patients With Advanced Non-Squamous Non-Small-Cell Lung Cancer. (2010) J Clin Oncol 28(9): 1527-1533. 
15. Kelley, Harris, Xie, et al. Preclinical studies to predict the disposition of Apo2L/tumor necrosis factor-related apoptosis-inducing ligand in humans: characterization of in vivo efficacy, pharmacokinetics, and safety. (2001) J Pharmacol Exp Ther 299(1): 31-38.

16. Ashkenazi, A., Pai, R., Fong, S., et al. Safety and antitumor activity of recombinant soluble Apo2 ligand. (1999) J Clin Invest 104(2): $155-162$.

17. van Engeland, Nieland, Ramaekers, et al. Annexin V-affinity assay: a review on an apoptosis detection system based on phosphatidylserine exposure. (1998) Cytometry 31(1): 1-9.

18. Boulares, H., Yakovlev, A., Ivanova, V., et al. Role of Poly(ADP-ribose) Polymerase (PARP) Cleavage in Apoptosis Caspase 3-Resistant PARP Mutant Increases Rates of Apoptosis in Transfected Cells. (1999) J Biol Chem 274(33): 22932-22940.

19. Kischkel, F., Lawrence, D., Chuntharapai, A., et al. Apo2L/ TRAIL-Dependent Recruitment of Endogenous FADD and Caspase-8 to Death Receptors 4 and 5. (2000) Immunity 12(6): 611-620.

20. Zhang, X., Franco, A., Nguyen, T., et al. Differential localization and regulation of death and decoy receptors for TNF-related apoptosis-inducing ligand (TRAIL) in human melanoma cells. (2000) J Immunol Baltim Md 1950 164(8): 3961-3970.

21. Daniels, R., Turley, H., Kierley, F., et al. Expression of TRAIL and TRAIL receptors in normal and malignant tissues. (2005) Cell Res 15(6): 430-438

22. Wang, Y., Tjandra, N. Structural insights of tBid, the caspase- 8 -activated Bid, and its BH3 domain. (2013) J Biol Chem 288(50): 3584035851 .

23. Rowinsky, E. Targeted induction of apoptosis in cancer management: the emerging role of tumor necrosis factor-related apoptosis-inducing ligand receptor activating agents. (2005) J Clin Oncol 23(36): 9394-9407.

24. Manzo, F., Nebbioso, A., Miceli, M., et al. TNF-related apoptosis-inducing ligand: Signalling of a "smart" molecule. (2009) Int J Biochem Cell Biol 41(3): 460-466.

25. Hymowitz, O'Connell, Ultsch, et al. A unique zinc-binding site revealed by a high-resolution X-ray structure of homotrimeric Apo2L/ TRAIL. (2000) Biochemistry 39(4): 633-640.

26. Cha, S.S., Kim, M.S., Choi, Y., et al. $2.8 \AA$ Resolution Crystal Structure of Human TRAIL, a Cytokine with Selective Antitumor Activity. (1999) Immunity 11(2): 253-261.

27. Kyrylkova, K., Kyryachenko, S., Leid, M., et al. Detection of apoptosis by TUNEL assay. (2012) Methods Mol Biol 887: 41-47.

28. Savitskaya, Onishchenko. Mechanisms of apoptosis. (2015) Biochemistry (Moscow) 80(11): 1393-1405.

29. Chawla-Sarkar, M., Bauer, J., Lupica, J., et al. Suppression of NF$\kappa B$ Survival Signaling by Nitrosylcobalamin Sensitizes Neoplasms to the Anti-tumor Effects of Apo2L/TRAIL. (2003) J Biol Chem 278(41): 39461-39469.
30. Zhang, L., Fang, B. Mechanisms of resistance to TRAIL-induced apoptosis in cancer. (2004) Cancer Gene Ther 12(3): 228-237.

31. Griffith, T.S., Chin, W.A., Jackson, G.C., et al. Intracellular regulation of TRAIL-induced apoptosis in human melanoma cells. (1998) J Immunol 161(6): 2833-2840.

32. Chawla-Sarkar., Bae., Reu., et al. Down regulation of Bcl-2, FLIP or IAPs (XIAP and survivin) by siRNAs sensitizes resistant melanoma cells to Apo2L/TRAIL-induced apoptosis. (2004) Cell Death \& Differ 11(8): 915-923.

33. Sun, S.Y., Yue, P., Zhou, J.Y., et al. Overexpression of Bcl2 Blocks TNF-Related Apoptosis-Inducing Ligand (TRAIL)-Induced Apoptosis in Human Lung Cancer Cells. (2001) Biochem Biophys Res Commun 280(3): 788-797.

34. Chawla-Sarkar, M., Leaman, D.W., Jacobs, B.S., et al. IFN-beta pretreatment sensitizes human melanoma cells to TRAIL/Apo2 ligand-induced apoptosis. (2002) J Immunol 169(2): 847-855.

35. Du, J., Wu, J., Fu, X., et al. Icariside II overcomes TRAIL resistance of melanoma cells through ROS-mediated down regulation of STAT3/ cFLIP signaling. (2016) Oncotarget 7(32): 52218-52229.

36. Prasad, S., Kim, J.H., Gupta, S.C., et al. Targeting death receptors for TRAIL by agents designed by Mother Nature. (2014) Trends Pharmacol Sci 35(10): 520-536.

37. Wiley, S., Schooley, K., Smolak, P., et al. Identification and characterization of a new member of the TNF family that induces apoptosis. (1995) Immunity 3(6): 673-682.

38. Ashkenazi, A. Targeting death and decoy receptors of the tumour-necrosis factor superfamily. (2002) Nature Reviews Cancer 2(6): $420-430$

39. Ashkenazi, A., Holland, P, Eckhardt, G. Ligand-Based Targeting of Apoptosis in Cancer: The Potential of Recombinant Human Apoptosis Ligand 2/Tumor Necrosis Factor-Related Apoptosis-Inducing Ligand (rhApo2L/TRAIL). (2008) J Clin Oncol 26(21): 3621-3630.

40. Luo, Q., Shen, Y.L., Wei, D.Z., et al. Optimization of culture on the overproduction of TRAIL in high-cell-density culture by recombinant Escherichia coli. (2006) Appl Microbiol Biotechnol 71(2):184-191.

41. Shen, Y.L., Zhang, Y., Sun, A.Y., et al. High-level production of soluble tumor necrosis factor-related apoptosis-inducing ligand (Apo2L/ TRAIL) in high-density cultivation of recombinant Escherichia coli using a combined feeding strategy. (2004) Biotechnology Letters 26(12): 981-984.

42. Wang, D., Shi, L. High-Level Expression, Purification, and In Vitro Refolding of Soluble Tumor Necrosis Factor-Related Apoptosis-Inducing Ligand (TRAIL). (2009) Appl Biochem Biotechnol 157(1): 1-9.
Ommega Online Publishers

Journal Title: International Journal of Cancer and Oncology (IJCO)

Journal Short Name: Int J Cancer Oncol
Journal ISSN: 2377-0902

E-mail: cancerandoncology@ommegaonline.com Website: www.ommegaonline.org 